\title{
The effects of a tea tree oil-containing gel on plaque and chronic gingivitis
}

\author{
S Soukoulis, ${ }^{*}$ R Hirsch*
}

\begin{abstract}
Background: This clinical study assessed the effects of topically applied tea tree oil (TTO)-containing gel on dental plaque and chronic gingivitis.

Methods: This was a double-blind, longitudinal, non-crossover study in 49 medically fit non-smokers (24 males and 25 females) aged 18-60 years with severe chronic gingivitis. Subjects were randomly assigned to three groups and given either TTO-gel (2.5 per cent), chlorhexidine (CHX) gel (0.2 per cent), or a placebo gel to apply with a toothbrush twice daily. Treatment effects were assessed using the Gingival Index (GI), Papillary Bleeding Index (PBI) and plaque staining score (PSS) at four and eight weeks.

Results: No adverse reactions to any of the gels were reported. The data were separated into subsets by tooth (anterior and posterior) and tooth surface (buccal and lingual). The TTO group had significant reduction in PBI and GI scores. However, TTO did not reduce plaque scores, which tended to increase over the latter weeks of the study period.

Conclusion: Although further studies are required, the anti-inflammatory properties of TTO-containing gel applied topically to inflamed gingival tissues may prove to be a useful non-toxic adjunct to chemotherapeutic periodontal therapy.

Key words: Essential oil, dental plaque, chronic gingivitis.

(Accepted for publication 1 March 2004.)
\end{abstract}

\section{INTRODUCTION}

Tea tree oil (TTO) is derived from the paper bark tea tree, which is part of the family Myrtaceae; it belongs to two genera, Leptospermum and Melaleuca. Australian Aborigines have used plant-derived medications including TTO for tens of thousands of years. These preparations are used both internally and externally for the alleviation of pain and the promotion of healing. ${ }^{1}$ TTO is used by Aborigines to treat abrasions, cuts, colds and influenza. TTO is now used around the world in many cosmetic, medicinal and dental products (e.g., 'natural' toothpastes). However,

"Dental School, The University of Adelaide. there is very little literature regarding the periodontal effects of TTO; such products do not have to undergo the strict evaluation procedures required by federal bodies for conventional products.

The main active ingredients of TTO are 1,8-cineole and terpinen-4-ol; it also contains many more components whose biological properties have not been thoroughly researched. These components are also found in other common essential oils, e.g., eucalyptus and fennel oil. 1,8-cineole has anti-inflammatory properties, ${ }^{2-4}$ and is able to penetrate human skin. ${ }^{5}$ Terpinen-4-ol has similar anti-inflammatory activities as 1,8 -cineol ${ }^{6,7}$ but also has anti-bacterial activity. ${ }^{8-12}$

TTO shares a similar range of antimicrobial activity with chlorhexidine (CHX), although their mechanisms of action differ., ${ }^{9218}$ They both have antibacterial, antiviral and antifungal properties. TTO has the potential to be a therapeutic agent in chronic gingivitis and periodontitis, conditions that have both bacterial and inflammatory components.

The aim of this study was to determine the effect of a TTO-containing gel used twice daily on existing gingival inflammation and plaque quantity in unsupervised, periodontally untreated subjects.

\section{MATERIAL AND METHODS}

The University of Adelaide's Human Research Ethics Committee gave ethical approval for the study. Informed consent was obtained from all subjects. Male and female non-smokers aged between 18-60 years with moderate to severe plaque-induced gingivitis (GI score of 2-3 in a minimum of one tooth in each quadrant) were recruited. The subjects had a minimum of 20 teeth and did not have diabetes, hepatic or kidney disease, rheumatoid arthritis, were not pregnant or lactating, had not received periodontal therapy in the last six months and had no known allergies to TTO. Other exclusion criteria included individuals taking steroids, NSAIDs, dilantin, antibiotics for at least seven days in the last six months and subjects requiring antibiotic cover for dental treatment.

The study design was double-blind, longitudinal non-crossover, using three groups; a test group, TTO

Australian Dental Journal 2004;49:2. 
Table 1. Distribution in treatment groups of subjects by age and gender

\begin{tabular}{lccccccccc}
\hline Group & Female & Male & $\mathrm{n}$ & Mean age & SD & LB 95\% CI & HB 95\% CI & Min age & Max age \\
\hline PLB & 7 & 8 & 15 & 46.53 & 7.90 & 42.16 & 50.91 & 32 & 61 \\
CHX & 10 & 8 & 18 & 44.89 & 11.62 & 39.11 & 50.67 & 26 & 63 \\
TTO & 7 & 9 & 16 & 45.50 & 8.84 & 40.79 & 50.21 & 30 & 62 \\
Total & 24 & 25 & 49 & 45.59 & 9.53 & 42.85 & 48.33 & 26 \\
\hline
\end{tabular}

(2.5 per cent TTO-containing gel), a positive control group, CHX (0.2 per cent chlorhexidine gel (Periogard, Colgate, NSW, Australia)), and a negative control group, PLB (placebo gel identical to the test agent but not containing TTO). The duration of the study was eight weeks with the following indices measured at 0 , four and eight weeks: Gingival Index (GI), ${ }^{19}$ Papillary Bleeding Index (PBI) and Plaque Surface Score (PSS). The plaque scoring system (PSS) was a modification by Turesky of the plaque index; ${ }^{20}$ disclosing solution containing 1 per cent w/v erythrosine (Disclogel, Colgate, NSW, Australia) was applied to the teeth.

At their first visit, subjects were instructed to apply the gel along the entire length of the supplied toothbrushes. Detailed instructions on the use of the gels were given to subjects in the form of a two page pamphlet. The gel was then to be used as a dentrifice making sure that it made contact with the gingival tissues adjacent to the teeth for a minimum of two minutes twice daily. No rinsing, eating or drinking was allowed for 30 minutes after gel application. Subjects were asked to refrain from using proprietary toothpastes and mouthwashes during the study and no auxiliary cleaning aids were allowed to be used during the course of the study. The gels were randomly distributed to the subjects; neither researcher nor subjects were aware of the contents of the tubes distributed.

Repeat examinations were carried out on five subjects between the ages of 26-63 years measuring GI, PBI, PSS at four sites around each tooth (one day between examinations) to establish intra-examiner error. The same examination procedures were used as in the subsequent study.

\section{Data analyses}

Statistical analyses of the data were carried out using a computer software programme (SPSS version 10.05 , Chicago, USA). ANOVA tests were used to identify significant differences between the means of the study groups. Scheffe's test was used to determine statistically significant variations between the means of each group. Finally, paired $t$ tests were used to assess the significance of changes within each group between time periods $0-4,4-8$ and $0-8$ weeks. The data for each subject were added, averaged and weighted depending on the number of teeth used from the subject.

\section{RESULTS}

\section{Profile of the study population}

Fifty-eight subjects were recruited, but due to several dropouts during the study, data from nine subjects were not used in the final analyses. The 49 subjects consisted of 25 males and 24 females. Table 1 shows the age and gender distribution; there were no statistically significant age or gender differences between the groups (Pearson chi-square, $\mathrm{p}=0.772$ and $\mathrm{p}=0.889$ respectively).

Statistical analysis showed that intra-examiner error was within acceptable limits.

There were no reports of adverse reactions to any of the gels. Few significant differences between the three groups were found in regards to mean changes in GI, PSS and PBI scores. When data for all teeth and surfaces were included in the analyses, most changes in all three groups occurred during the first four weeks of the study. When the data were separated into anterior/ posterior and buccal/lingual surfaces, ANOVA tests of PBI showed that the subjects using the TTO-containing gel had statistically significant means changes compared to the positive and negative controls in posterior teeth and buccal surface data sets. ANOVA tests were also determined for the differences between groups in terms of improvement, i.e., lower scores in weeks four and eight compared to baseline, for PBI, GI and PSS data. See Fig 1 and 2 for a summary of PBI and GI data.

The TTO group showed the most number of improvements in PBI in data sets for all teeth and surfaces (grouped data), buccal, anterior and posterior teeth. All but one of the data sets (anterior teeth data set) was statistically significant. The buccal data showed the largest improvement in PBI scores. The TTO group had the greatest number of time periods with the most improvements (9), next was the CHX group (4) and then the PLB group (1).

The CHX group had the greatest number of time periods with the most improvements in GI (7), next was the TTO group (6) and then the PLB group (5). The TTO group had the greatest number of improved sites in GI. Overall the differences between the groups were not great, indicated by the lack of statistically significant results.

There were no statistically significant differences in PSS between the groups. The TTO group consistently had the lowest number of sites that improved for all time periods. The CHX group generally had more improvement in PSS than the other groups. For example, the CHX group had more time periods with the most improvements (11), next was the PLB group (4) and then the TTO group (1).

\section{Mean changes within groups}

Paired $t$ tests showed that changes in GI and PBI were statistically significant (see Fig 1 and 2). The 

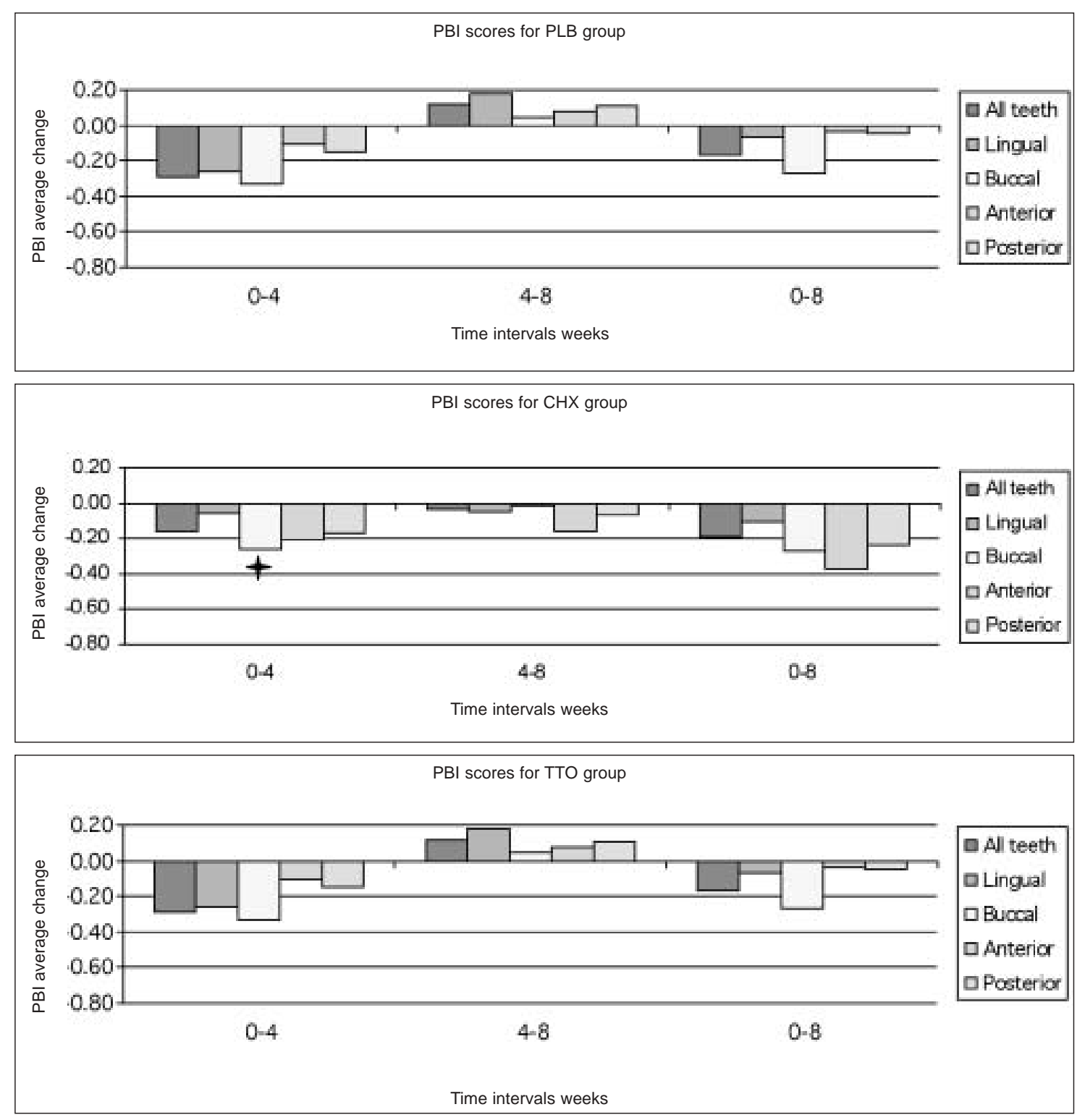

Fig 1. Mean changes in PBI of all teeth, buccal/lingual and anterior/posterior data sets.

( + denotes statistically significant intragroup changes between time periods $0-4,4-8$ and $0-8$ weeks).

majority of these significant changes occurred in the TTO and CHX groups. Paired $t$ tests confirmed that the use of TTO-containing gel resulted in the greatest change in PBI for all data sets during weeks $0-4$ and $0-8$. This occurred in 10 time periods out of 15 (three time periods, i.e., weeks $0-4,4-8$ and $0-8$ for five data sets). Except for lingual surfaces, all of these results were statistically significant. The group that used a CHX-containing gel had five time periods with the greatest change, but none were statistically significant (Fig 1). The CHX group had the greatest change in GI in many data sets (eight time periods). Of these, seven were statistically significant. The TTO group was next with six time periods with the greatest change, of these five were statistically significant (Fig 1).

No significant changes were observed in PSS. However, the TTO group was the only group to show an increase in plaque scores for weeks $0-4$ and $0-8$. The $\mathrm{CHX}$ group had the greatest reduction in plaque scores in many data sets (7), the placebo was next (6), and the TTO group had the least (1).

\section{DISCUSSION}

Within the limitations of the study, it was apparent that TTO-containing gel decreased the level of gingival

Australian Dental Journal 2004;49:2. 

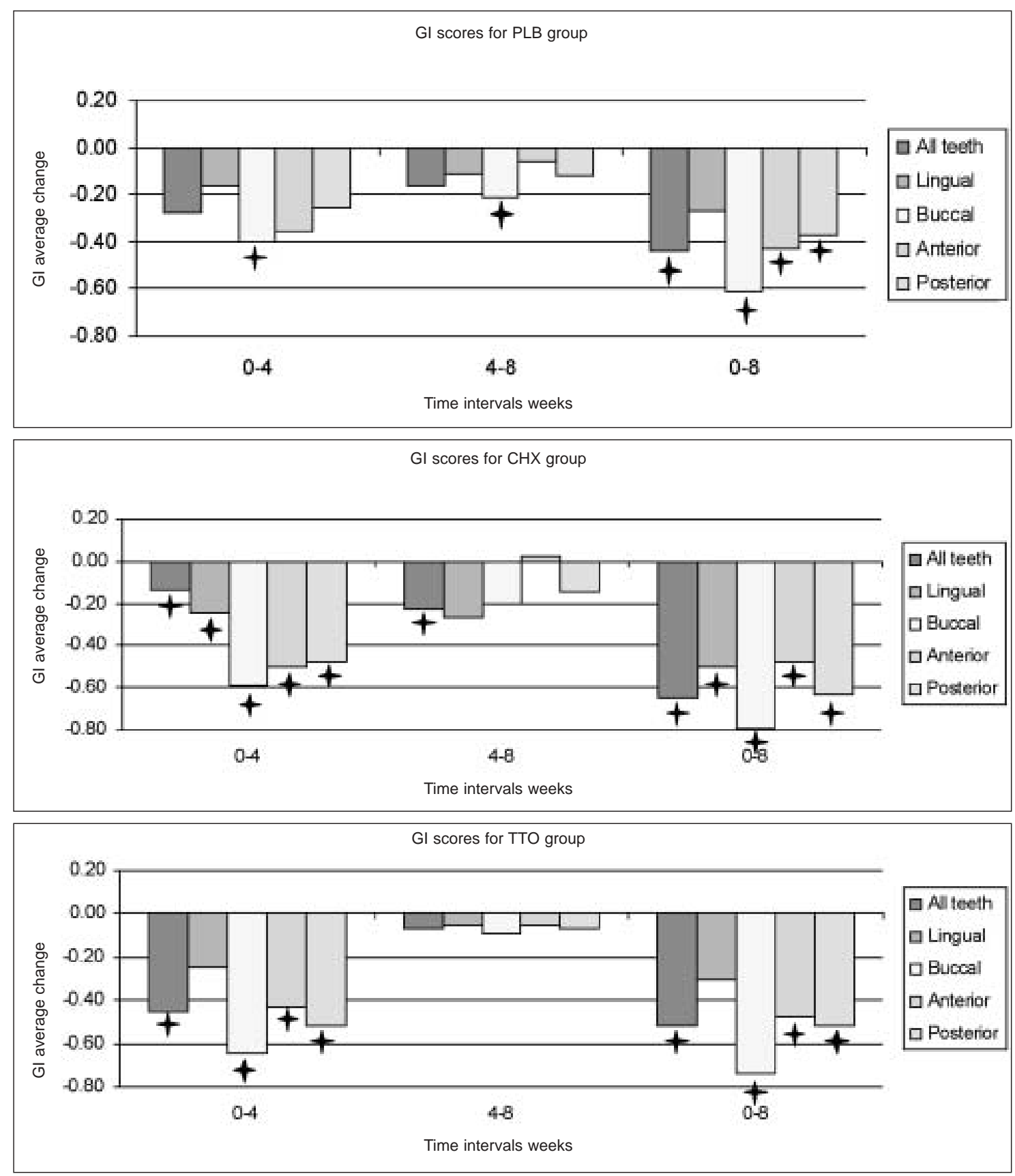

Fig 2. Changes in GI of all teeth, buccal/lingual and anterior/posterior data sets.

inflammation more so than did the positive and negative controls. Of particular interest, the improvements in PBI in the TTO group were not accompanied by reduced plaque scores.

Although the TTO group did not exhibit mean changes greater than one unit, it did approach it for several data sets. For example, it reached a mean value of 0.75 for buccal surfaces (average change weeks 0-4),

Australian Dental Journal 2004;49:2 three times that achieved by the CHX group (0.26) and over twice that of the PLB group (0.33). The 95 per cent confidence intervals for buccal surfaces showed a one unit change (range 0.49-1.01), indicating that clinically measurable changes occurred at buccal sites in the TTO group.

The TTO group had the largest number of improved sites for the following data sets: all teeth, posterior 
teeth, anterior teeth and buccal surfaces. All data sets (excluding the anterior teeth) had statistically significant improvements for the 0-4 week time period. The buccal surfaces showed the largest improvements in PBI. The changes observed in PBI indicated that TTO reduced existing gingivitis in subjects to a greater extent than occurred in the other groups.

No significant differences between group means or mean changes were detected in the ANOVA tests, indicating that the responses were similar or small, depending on the data set. The results are similar to those of most CHX gel studies ${ }^{21-23}$ but the magnitude of changes reported by these authors was greater than observed in the current study. A likely explanation is that subjects had a scale and clean at baseline in the studies referred to. The changes reported were therefore due to a combined effect of mechanical treatment and CHX gel. The finding in this study that the TTO group and the CHX groups had decreased PBI and GI scores may point to a clinically relevant therapeutic effect because oral hygiene practices were average to poor and subjects had considerable deposits of supragingival/subgingival plaque and calculus throughout the study.

There is no 'gold standard' index for assessing the degree of gingivitis or the treatment effects of various agents or treatment methods. Since the GI has been the most widely used index in studies investigating oral hygiene products, it was included in this study to permit comparison between studies. However, there are significant limitations inherent in the GI that receive scant attention in the periodontal literature. GI has fewer scoring criteria than PBI; it therefore provides less information and is less sensitive than the PBI to smaller changes in gingival inflammation. The GI was formulated when the science of periodontology was in its infancy. At the time, it was thought that colour changes in the gingival tissues preceded the development of BOP, whereas subsequent research has shown the reverse to be the case. ${ }^{24,25}$ Gingival colour change is not necessarily an accurate indicator of gingival inflammation, as incipient gingival inflammatory lesions have BOP but do not necessarily have altered colour. Also mucocutaneous lesions (erosive lichen planus, pemphigus, etc.) of the gingival tissues can be a confounding factor when using colour changes in periodontal indices.

If the current study were to be extended, the inclusion of a clinical measure of gingival inflammation that increased objectivity would be warranted. This may include an assessment of subgingival pocket temperatures ${ }^{26}$ or cytokine measurements (e.g., IL-1, IL6, IL-8). ${ }^{27}$

Information gathered from the current trial and the limited literature indicates that TTO's antibacterial effects, although potent in vitro, do not translate to inhibition of plaque formation in vivo. Few changes occurred in the PSS parameter, including in the CHX group; no changes in PSS reached statistical significance. There are only two published studies assessing TTO's effects on oral bacteria and on plaque formation in vivo. The response in PSS of the TTO group in the current trial was similar to other studies that have investigated TTO as an oral antibacterial agent. ${ }^{28}$ However, direct comparison of the results is not possible because the TTO vehicle in those investigations was a mouthwash rather than a gel and the studies' protocols were very different. A TTO containing mouthwash was effective in decreasing salivary bacterial counts for approximately $1 / 2$ an hour. This decrease was comparable to the CHX control, but after six hours, salivary bacterial levels nearly returned to normal whereas the activity of $\mathrm{CHX}$ persisted. Similar results were seen in a clinical trial of TTO mouthwash, ${ }^{28}$ in a study (crossover design) with a washout period of three days in eight subjects. In the first week, water was used as a mouthwash in the absence of oral hygiene for four days, CHX mouthwash was used in the second week and TTO mouthwash in the last week. Plaque index and areas were scored, as was vital fluorescence to detect vital bacterial colonies. The TTO containing mouthwash had no effect on the quantity or quality of supragingival plaque.

The CHX group's plaque scores in this study did not change significantly. However, the CHX group had the greatest mean changes compared to the other groups.

This is contrary to the findings of clinical studies investigating CHX gel, although clinical effects of CHX gel on plaque have been variable depending on the frequency of use, concentration used and whether or not subjects were treated or untreated at the beginning of the study making comparison between studies difficult. ${ }^{21-23,29,30}$ Consistent with the present findings, the majority of treatment effects occurred early in the observation periods (0-4 weeks), possibly indicating decreased compliance later in the studies or that the end point of treatment effects had been reached, i.e., improvements in clinical parameters reached a plateau after which no further improvement was possible. ${ }^{21,22}$

Poor compliance with gel use may have contributed to the limited changes seen in PSS, particularly in relation to the CHX group. An indirect indicator of compliance may have been the time taken by subjects to use the gel over four weeks. The use of gel varied greatly. The majority of subjects claimed that the amount of gel supplied was sufficient for four weeks, with little gel remaining. A few subjects reported that they had a lot of gel remaining after four weeks, possibly indicating poorer compliance.

Subjects in this study had pre-existing plaque and supragingival/subgingival calculus deposits that made effective oral hygiene difficult and probably facilitated plaque growth. Using disclosing solution on teeth with significant calculus deposits would result in staining irrespective of plaque formation, confounding plaque measurements. This would have affected all groups' measurements.

Australian Dental Journal 2004:49:2. 


\section{Possible periodontal applications of TTO gel}

Since decreased gingival inflammation occurred in subjects who used TTO without a concomitant decrease in plaque scores, its mechanism of activity could have been anti-inflammatory rather than antibacterial. The components of TTO are known to decrease inflammation in in vitro and in vivo settings. ${ }^{2,3,6,7}$ For example, subjects who were administered Soledum ${ }^{\circledR}$ (containing 1,8-cineole) showed a significant decrease in the production of $\mathrm{LBT}_{4}$ and $\mathrm{PGE}_{2}$ in isolated monocytes. ${ }^{2}$ 1,8-cineole has also been shown to significantly inhibit in vitro cytokine production (TNF-a, IL-1b) from LPS stimulated monocytes. ${ }^{3}$ Similar in vitro effects were shown on monocytes by terpinen-4-ol. ${ }^{6,7}$

The components of TTO are known to have lipophilic properties which facilitate its diffusion through epithelium. ${ }^{5}$ If TTO is readily absorbed after topical application into the gingival tissues and has anti-inflammatory properties once it has entered the connective tissues, it would be a unique, non-toxic agent that would be a useful addition to the current range of chemotherapeutic periodontal treatment options.

\section{REFERENCES}

1. Barr A, Chapman J, Smith N, Beveridge M. Traditional bush medicines - An Aboriginal pharmacopoeia. Victoria: Greenhouse Publications, 1988.

2. Juergens UR, Stober M, Schmidt-Schilling L, Kleuver T, Vetter H Antiinflammatory effects of eucalyptol (1.8-cineole) in bronchia asthma: inhibition of arachidonic acid metabolism in human blood monocytes ex vivo. Eur J Med Res 1998;3:407-412.

3. Juergens UR, Stober M, Vetter H. Inhibition of cytokine production and arachidonic acid metabolism by eucalyptol (1.8 cineole) in human blood monocytes in vitro. Eur J Med Res 1998;3:508-510.

4. Santos FA, Rao VS. Antiinflammatory and antinociceptive effects of 1,8-cineole a terpenoid oxide present in many plant essentia oils. Phytother Res 2000;14:240-244

5. Williams AC, Barry BW. Terpenes and the lipid-proteinpartitioning theory of skin penetration enhancement. Pharm Res 1991;8:17-24.

6. Hart PH, Brand C, Carson CF, Riley TV, Prager RH, Finlay-Jones JJ. Terpinen-4-ol, the main component of the essential oil of Melaleuca alternifolia (tea tree oil), suppresses inflammatory mediator production by activated human monocytes. Inflamm Res 2000;49:619-626.

7. Brand C, Ferrante A, Prager RH, et al. The water soluble components of the essential oil of Melaleuca alternifolia (tea tree oil) suppress the production of superoxide by human monocytes, but not neutrophils, activated in vitro. Inflamm Res 2001;50:213-219.

8. Carson CF, Cookson BD, Farrelly HD, Riley TV. Susceptibility of methicillin-resistant Staphylococcus aureus to the essential oil of Melaleuca alternifolia. J Antimicrob Chemother 1995;35:421424.

9. Carson CF, Riley TV. Antimicrobial activity of the major components of the essential oil of Melaleuca alternifolia. J Appl Bacteriol 1995;78:264-269.

10. Hammer KA, Carson CF, Riley TV. In vitro susceptibility of Malassezia furfur to the essential oil of Melaleuca alternifolia. J Med Vet Mycol 1997;35:375-377.

11. Hammer KA, Carson, CF, Riley TV. In-vitro activity of essential oils, in particular Melaleuca alternifolia (tea tree) oil and tea tree oil products, against Candida spp. J Antimicrob Chemother 1998;42:591-595.

Australian Dental Journal 2004:49:2.
12. May J, Chan CH, King A, Williams L, French GL. Time-kill studies of tea tree oils on clinical isolates. J Antimicrob Chemother 2000;45:639-643.

13. Moran J, Addy M, Newcombe R. A clinical trial to assess the efficacy of sanguinarine-zinc mouthrinse (Veadent) compared with chlorhexidine mouthrinse (Corsodyl). J Clin Periodontol 1988;15:612-616.

14. Ostela I, Tenovuo J. Antibacterial activity of dental gels containing combinations of amine fluoride, stannous fluoride, and chlorhexidine against cariogenic bacteria. Scand J Dent Res 1990;98:1-7.

15. Shapiro S, Meier A, Guggenheim B. The antimicrobial activity of essential oils and essential oil components towards oral bacteria. Oral Microbiol Immunol 1994;9:202-208.

16. Cox SD, Gustafson JE, Mann CM, et al. Tea tree oil causes $\mathrm{K}^{+}$ leakage and inhibits respiration in Escherichia coli. Lett Appl Microbiol 1998;26:355-358.

17. Cox SD, Mann CM, Markham J, et al. The mode of antimicrobial action of the essential oil of Melaleuca alternifolia (tea tree oil). J Appl Microbiol 2000;88:170-176.

18. Fine DH, Furgang D, Barnett ML, Drew C, Steinberg L, Charles $\mathrm{CH}$, Vincent JW. Effect of an essential oil-containing antiseptic mouthrinse on plaque and salivary Streptococcus mutans levels. J Clin Periodontol 2000;27:157-161.

19. Löe H. The Gingival Index, the Plaque Index and the Retention Index Systems. J Periodontol 1967;38:610-616.

20. Fischman SL. Current status of indices of plaque. J Clin Periodontol 1986;13:371-374.

21. Hoyos DF, Murray JJ, Shaw L. The effect of chlorhexidine gel on plaque and gingivitis in children. Br Dent J 1977;142:366-369.

22. Joyston-Bechal S, Smales FC, Duckworth R. Effect of metronidazole on chronic periodontal disease in subjects using a topically applied chlorhexidine gel. J Clin Periodontol 1984;11:53-62.

23. Lie T, Enersen M. Effects of chlorhexidine gel in a group of maintenance-care patients with poor oral hygiene. J Periodontol 1986;57:364-369.

24. Meitner SW, Zander HA, Iker HP, Polson AM. Identification of inflamed gingival surfaces. J Clin Periodontol 1979;6:93-97.

25. Hirsch RS, Clarke NG, Townsend GC. The effect of locally released oxygen on the development of plaque and gingivitis in man. J Clin Periodontol 1981;8:21-28.

26. Wolff LF, Koller NJ, Smith QT, Mathur A, Aeppli D. Subgingival temperature: relation to gingival crevicular fluid enzymes, cytokines, and subgingival plaque micro-organisms. J Clin Periodontol 1997;24:900-906.

27. McGee JM, Tucci MA, Edmundson TP, Serio CL, Johnson RB. The relationship between concentrations of proinflammatory cytokines within gingiva and the adjacent sulcular depth. J Periodontol 1998;69:865-871.

28. Arweiler NB, Donos N, Netuschil L, Reich E, Sculean A. Clinical and antibacterial effect of tea tree oil - a pilot study. Clin Oral Investig 2000;4:70-73.

29. Hansen F, Gjermo P, Eriksen HM. The effect of a chlorhexidinecontaining gel on oral cleanliness and gingival health in young adults. J Clin Periodontol 1975;2:153-159.

30. Lennon MA, Davies RM. A short-term evaluation of a chlorhexidine gel on plaque deposits and gingival status. Pharmacol Ther Dent 1975;2:13-19.

Address for correspondence/reprints: Dr Robert Hirsch Dental School

The University of Adelaide South Australia 5005

Email: robert.hirsch@adelaide.edu.au 